\title{
ALPHA-PHOTON COINCIDENCE SPECTROSCOPY ALONG ELEMENT 115 DECAY CHAINS*
}

\section{Rudolpha ${ }^{\mathrm{a}}$, U. Forsberg $^{\mathrm{a}}$, P. Golubev ${ }^{\mathrm{a}}$, L.G. SARmiento ${ }^{\mathrm{a}}$} A. YAKusheV ${ }^{b}$, L.-L. Andersson ${ }^{c}$, A. Di NitTo ${ }^{d}$ Ch.E. Düllmann ${ }^{b, c, d}$, J.M. Gates ${ }^{\mathrm{e}}$, K.E. Gregorich ${ }^{\mathrm{e}}$, C.J. Gross ${ }^{\mathrm{f}}$ R.-D. HerzberG ${ }^{\text {g }}$, F.P. Hessberger ${ }^{\text {b,c }}$, J. KhuyagbaAtar ${ }^{c}$ J.V. KRATZ ${ }^{\mathrm{d}}$, K. RYKACZEWSKI ${ }^{\mathrm{f}}$, M. SCHÄDEL ${ }^{\mathrm{b}, \mathrm{h}}, \mathrm{S} . \AA_{B \mathrm{BERG}}^{\mathrm{a}}$ D. Ackermann ${ }^{b}$, M. Block ${ }^{b}$, H. Brand ${ }^{b}$, B.G. Carlsson ${ }^{\mathrm{a}}$, D. Cox ${ }^{\mathrm{g}}$

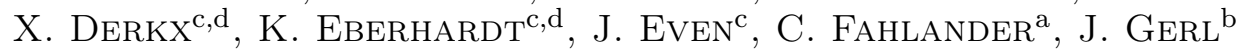
E. JÄGER ${ }^{\mathrm{b}}$, B. Kindler ${ }^{\mathrm{b}}$, J. KRIER ${ }^{\mathrm{b}}$, I. Kojouharov ${ }^{\mathrm{b}}$, N. Kurz ${ }^{\mathrm{b}}$

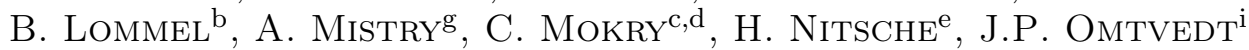
P. PAPADAKIS ${ }^{g}$, I. RAGNARSSON ${ }^{\mathrm{a}}$, J. Runke ${ }^{\mathrm{b}}, \mathrm{H}$ SCHAFFnER $^{\mathrm{b}}$

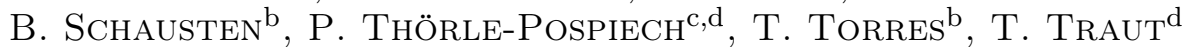
N. Trautmann ${ }^{d}, A \cdot$ TÜrler $^{j}, A \cdot$ WARD $^{g}, D^{2} \cdot$ E. WARD $^{a}, N \cdot$ Wiehl $^{c, d}$

${ }^{a}$ Lund University, Lund, Sweden

${ }^{\mathrm{b}}$ GSI Helmholtzzentrum für Schwerionenforschung GmbH, Darmstadt, Germany ${ }^{\mathrm{c}}$ Helmholtz Institute Mainz, Mainz, Germany ${ }^{\mathrm{d} J o h a n n e s ~ G u t e n b e r g-U n i v e r s i t a ̈ t ~ M a i n z, ~ M a i n z, ~ G e r m a n y ~}$

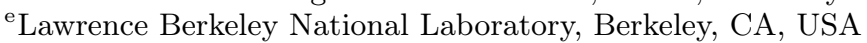

${ }^{\mathrm{f}}$ Oak Ridge National Laboratory, Oak Ridge, TN, USA

${ }^{\mathrm{g}}$ University of Liverpool, Liverpool, United Kingdom

${ }^{\mathrm{h}}$ Advanced Science Research Center, Japan Atomic Energy Agency, Tokai, Japan ${ }^{\mathrm{i}}$ University of Oslo, Oslo, Norway

${ }^{\mathrm{j}}$ Paul Scherrer Institute and University of Bern, Villigen, Switzerland

\section{(Received November 25, 2013)}

Produced in the reaction ${ }^{48} \mathrm{Ca}+{ }^{243} \mathrm{Am}$, thirty correlated $\alpha$-decay chains were observed in an experiment conducted at the GSI Helmholzzentrum für Schwerionenforschung, Darmstadt, Germany. The decay chains are basically consistent with previous findings and are considered to originate from isotopes of element 115 with mass numbers 287, 288, and 289. A set-up aiming specifically for high-resolution charged particle and photon coincidence spectroscopy was placed behind the gas-filled separator TASCA. For the first time, $\gamma$ rays as well as X-ray candidates were observed in prompt coincidence with the $\alpha$-decay chains of element 115 .

DOI:10.5506/APhysPolB.45.263

PACS numbers: 21.10.-k, 23.20.Lv, 23.60+e, 27.90.+b

* Presented at the XXXIII Mazurian Lakes Conference on Physics, Piaski, Poland, September 1-7, 2013. 


\section{Introduction}

On the cruise to the long-sought 'Island of Stability' [1], collaborations working at the Flerov Laboratory for Nuclear Research in Dubna, Russia, have landmarked about 50 new superheavy isotopes on the nuclidic chart $[2,3]$. These isotopes are interpreted to include elements with proton numbers $Z=113-118$ [4], produced via ${ }_{20}^{48} \mathrm{Ca}$-induced fusion-evaporation reactions on radioactive actinide targets of $\mathrm{Np}, \mathrm{Pu}, \mathrm{Am}, \mathrm{Cm}, \mathrm{Bk}$, and $\mathrm{Cf}$ $(Z=93-98)$.

Following these first expeditions, confirming evidence for some of the heaviest elements was put forward [5-11]. This led to the official acknowledgment of the discovery of the elements $Z=114, \mathrm{Fl}$, and $Z=116, \mathrm{Lv}$, by the IUPAC/IUPAP Joint Working Party [12]. Additional experimental work to support claims on the discovery of elements $Z=113,115$, and 118 was requested [12], not least because, so far, only indirect techniques were applied to assign $Z$ of these superheavy elements.

The $\alpha$ decay of odd $-A$ and odd-odd nuclei typically proceeds into excited states in the daughter nucleus [13, 14], which often implies complex $\alpha$-decay pattern. While this feature can severely hamper indirect means of assigning $Z$ and $A$, it opens the possibility to 'fingerprint' $Z$ by means of characteristic X-rays [15]. Correspondingly, a high-resolution $\alpha$-photon coincidence spectroscopy experiment was conducted to investigate decay chains supposedly originating from element 115 [16]. Here, we focus on details of the Ge-detector analysis, further Monte-Carlo simulations, and assessments of the hitherto available published data on the isotopes ${ }^{287,288} 115$ [16-18].

\section{Experimental}

Our experiment was conducted at the GSI Helmholtzzentrum für Schwerionenforschung in Darmstadt, Germany. We have studied the nuclear reaction ${ }^{48} \mathrm{Ca}+{ }^{243} \mathrm{Am}$ at two beam energies, both close to the maximum cross section of the anticipated $3 n$-evaporation channel leading to ${ }^{288} 115$ [18]. A beam integral of about $6 \times 10^{18}$ was collected over a period of three weeks with a typical beam intensity of $6 \times 10^{12}{ }^{48} \mathrm{Ca}$ ions per second. This intensity is time averaged over the pulsed structure of the UNILAC ( $5 \mathrm{~ms}$ beam on, $15 \mathrm{~ms}$ beam off). At the entrance of the recoil separator TASCA [10, 19, 20] the beam particles impinged on one out of four target segments. They had an average thickness of $0.83(1) \mathrm{mg} / \mathrm{cm}^{2}{ }^{243} \mathrm{Am}_{2} \mathrm{O}_{3}$, which was electroplated onto 2.20(5) $\mu \mathrm{m}$ thick Ti backing foils [21]. TASCA was filled with He-gas at $p_{\mathrm{He}}=0.8$ mbar and set to center ions with a magnetic rigidity of $B \rho=2.21 \mathrm{Tm}$ in the focal plane for most of the experiment [22]. These ions entered the multi-detector spectroscopy set-up TASISpec [23]. 
The heart of TASISpec is a cube-like arrangement of five $32 \times 32$-strip double-sided silicon strip detectors (DSSSD), i.e. TASISpec is fully pixelized. The $n$-doped sides of the DSSSDs were processed with standard spectroscopy electronics [23]. The preamplified signals of all $p$-doped sides were digitized as $70-\mu \mathrm{s}$ long traces by $60 \mathrm{MHz}, 12$-bit sampling ADCs. To detect photons, $\mathrm{X}$ rays or $\gamma$ rays, five large, composite germanium detectors were placed closely behind the five DSSSDs. They were operated in coincidence with charged-particle decays registered by the DSSSDs. The signals of in total 25 individual germanium detector crystals were handled by three commercial SIS3302 100-MHz, 16-bit sampling ADCs. The data acquisition was triggered by a coincident signal from a $p$-side and $n$-side strip of the implantation detector. Time-averaged ( $c f$. pulsed UNILAC beam structure) particle trigger rates were typically 100-120 events per second.

Due to the rather high radiation levels inside the TASCA cave - in particular during beam-on periods - and despite several passive means to reduce the $\gamma$-ray background, each Ge-detector crystal runs at rates significantly beyond $10^{4} / \mathrm{s}$. This number is generally considered as upper limit for decent signal processing with analog electronics; the baselines of the preamplified signals become more and more unstable, and there is an increasing rate of pile-up events. Thus, for this type of experiments, it is mandatory to use sampling ADC electronics. First of all, the FPGA software of the modules, which analyses the traces on-line, sets pile-up flags. Such events are strictly disregarded in the offline analysis, leading in practice to a reduced overall photon detection efficiency during beam-on periods. Secondly, both baseline position and flat-top energies of pure Ge-detector traces are recorded. Some details of the Ge-detector analysis are illustrated in Fig. 1. Panels (a) and (b) refer to uncorrected and panels (c) and (d) to baselinecorrected Ge-detector energy spectra. Clearly, this offline correction is a necessary prerequisite to retain proper spectral quality. One can note that different types of Ge-crystals and preamplifiers yield different baseline dependencies, which can be readily taken care of by studying and properly correcting two-dimensional spectra like the one in Fig. 1 (a).

Last but not least, it turns out that the Ge-energy spectra arising from baseline values above the dashed line in Fig. 1(c) are 'white', eventually because the SIS3302 firmware algorithm fails at very high rates. Disregarding these events, an improved peak-to-total ratio is achieved, which can be viewed in panels (e) and (f) by comparing the spectra in black (all data) and gray ('white' background removed). The spectra in Fig. 1 (e) are taken at beam-on or beam-off periods, and no prompt coincidence with an event trigger is required. This gives rise to a number of peaks with sufficient yield to perform yet another fine tune of Ge-detector data. Using the $139.7-\mathrm{keV}$ line from ${ }^{74} \mathrm{Ge}(n, \gamma)$ reactions together with the $511.0-\mathrm{keV} e^{+} e^{-}$annihi- 
lation peak, linear gain re-calibrations can be done for each Ge-detector crystal and for in total about ten sub-sets of data. This long-term correction leads to a further noticeable improvement of the overall energy resolution of the TASISpec Ge-detector system.
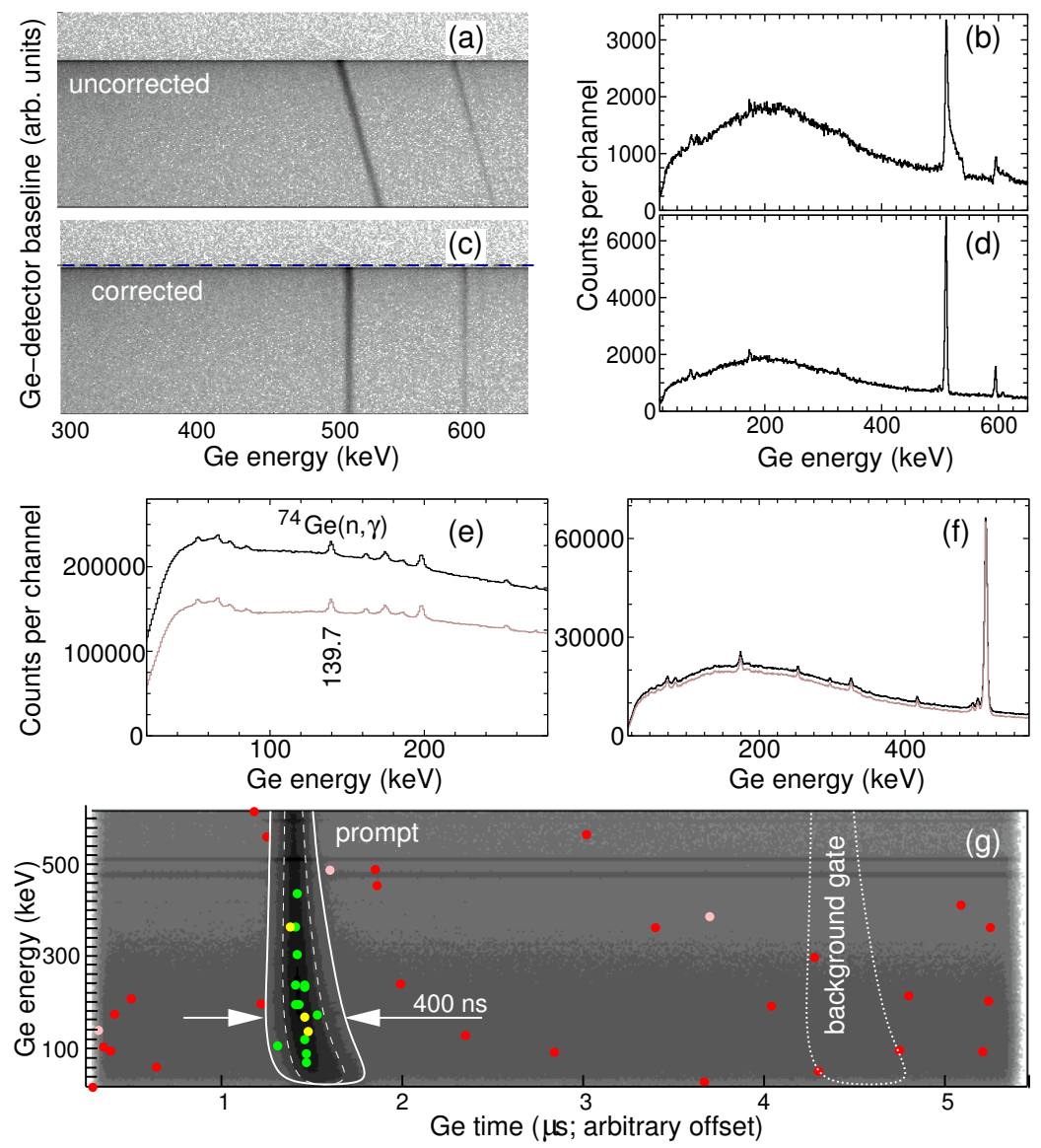

Fig. 1. Color on-line: Panels (a) and (c) provide uncorrected and corrected twodimensional plots of baseline vs. energy for the central Ge detector of the cluster detector positioned behind the TASISpec implantation DSSSD [23, 27]. Spectra in panels (b) and (d) are the corresponding projections. Data stems from both beamon and beam-off periods. Panels (e) and (f) show sum spectra during beam-on and beam-off periods without a prompt coincidence condition, and only during beam-off and with a prompt coincidence requirement, respectively. The gray spectra require the Ge-detector baseline being below the dashed line in panel (c). Panel (g) provides the correlation between Ge-detector energies and Ge-detector times measured with respect to the event trigger, i.e. an energy-corrected timing signal from the implantation DSSSD, during beam-off periods. See the text for more details. 
Figure 1 (f) shows total projections during beam-off periods in prompt coincidence with the event trigger. In the two-dimensional plot in panel $(\mathrm{g})$, the prompt (background) coincidence condition is illustrated by the full (dotted) white line. Since the event trigger arises from the implantation DSSSD, its timing depends on the energy of the registered particle in the DSSSD. Hence, the Ge-detector timing relative to the event trigger can be and has been corrected for this effect as well.

Inside the prompt coincidence window, which amounts to some generous 300-400 ns over the energy range of interest, there are 16 prompt $\alpha$-photon coincidences associated with the 22 decay chains of ${ }^{288} 115$ ( $c f$. Sec. 3); 13 relate to full-energy or fully reconstructed $\alpha$-decay energies (white/green circles) and 3 are linked to escaped $\alpha$ particles (dark gray/yellow). The remaining regime of the $5 \mu$ s measurement window contains 29 more random (or delayed!) coincidences; 26 with full-energy or fully reconstructed $\alpha$ decays (black/red) and 3 linked to escaped $\alpha$ particles (light gray/pink). As $29 \times 350 \mathrm{~ns} / 5000 \mathrm{~ns} \sim 2$, one expects on average two random coincidences inside the prompt window. This average can be reduced to half by using the, in fact, more realistic window indicated by the dashed white line. One green circle may become a random coincidence: it turns out to be the (uninteresting) single 105-keV photon of the first decay step ( $c f$. Fig. 2 in Ref. [16]). As stated in [16], yet another "random photon may arise from an unrelated escapelike event possibly being assigned as a true member to any of the present 22 chains".

More details on the experiment and other parts of the analysis procedures can be found in Refs. [16, 22, 24, 25].

\section{Selected results}

In three weeks of beamtime, a total of 30 correlated $\alpha$-decay chains of non-random origin were registered [16, 25], produced with an overall cross section of some $10 \mathrm{pb}$, which is compatible with results in Ref. [18]. Measured decay properties such as $\alpha$-particle energies, lifetimes, and decay modes of 5 - $\alpha$-decay-long chains associated with the $3 n$-(22 chains) and $4 n$-(1 chain) evaporation channels, respectively, are in agreement with those 31 and 2 chains recently presented in Ref. [18]. This leads us to conclude that we have observed the decay sequences of the same nuclei as were formerly observed by Oganessian et al. Collecting and averaging all data published so far gives rise to the two decay sequences shown in Fig. 2.

One example of the congruence of the data of Refs. [16, 18] is illustrated in Fig. 3, which provides correlation times of the $5 \alpha$-decay steps and the spontaneous fission along the chains associated with ${ }^{288} 115$. The spectra in dashed (red) relate to Ref. [18], the spectra in solid (black) refer to the 


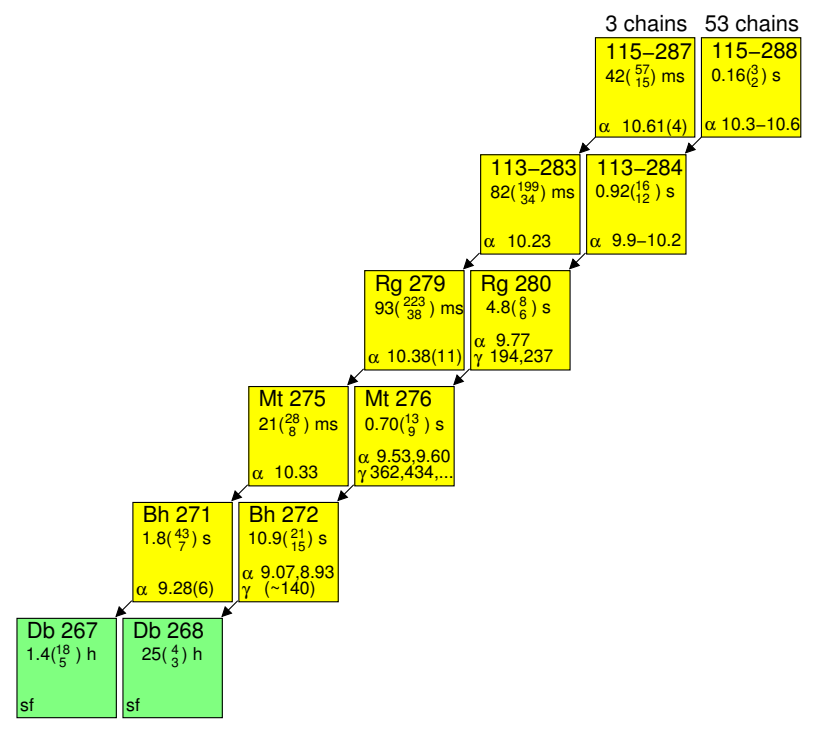

Fig. 2. Color on-line: Proposed decay chains of ${ }^{287} 115$ and ${ }^{288} 115$ based on hitherto accumulated published data [16-18]. Half-lives, $T_{1 / 2}$, are provided with uncertainties (cf. Fig. 3). $\alpha$-decay energies, $E_{\alpha}$, are given in $\mathrm{MeV}$. Their uncertainties are included if in excess of $10 \mathrm{keV}$. Only a range of $\alpha$-decay energies can be deduced for ${ }^{288} 115$ and ${ }^{284} 113$, respectively (see Fig. 4). $\gamma$-ray energies, $E_{\gamma}$, are in $\mathrm{keV}$.

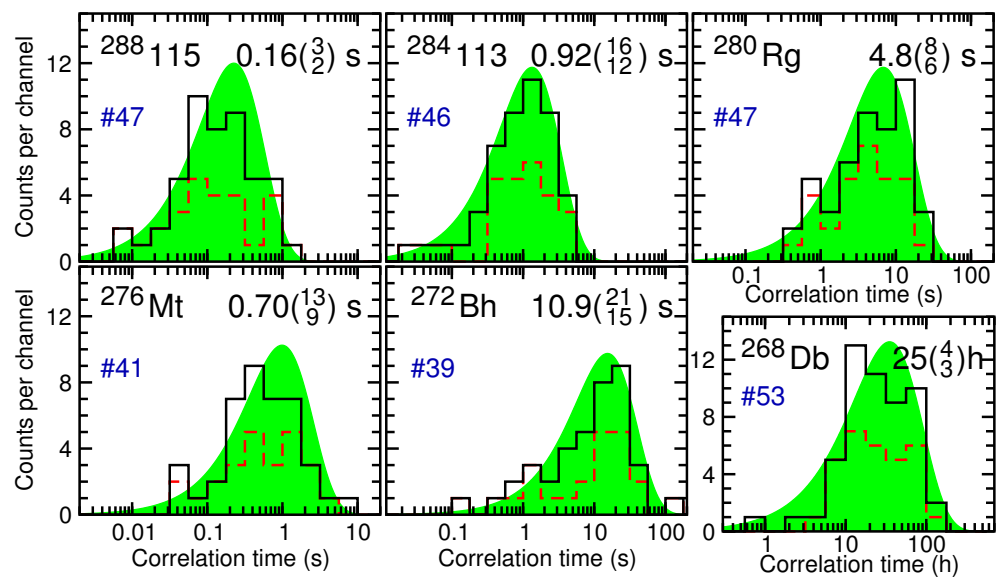

Fig. 3. Color on-line: Correlation times along the decay chains starting with the isotope ${ }^{288} 115$ based on published data from Ref. [18] (dashed/red lines). The solid lines (black) correspond to the combined experimental data of Refs. [16, 18]. Each panel is labeled with the decaying isotope. The number behind the hashtag, \#, refers to the number of data points available to derive the half-life of a given decay step (continuous gray/green distributions) [26]. 
sum spectra of Refs. [16, 18]. The number $N$ of firm data points, i.e. those with proper correlations with preceding $\alpha$ decays, is provided by $\# N$ in each panel. Due to the apparently much longer half-life of ${ }^{268} \mathrm{Db}$, this restriction has been relaxed for this isotope. The experimental spectra are underlayed with smooth distributions (gray/green), which correspond to decay-time distributions expected for decays with the half-lives indicated in the upper right corner of each panel [26]. With the present statistics, it is difficult to argue in favor of different decay branches with differing half-lives in any given step of the anticipated ${ }^{288} 115$ decay chain.

High-resolution spectroscopy data for $\alpha$-photon coincidences of the ${ }^{288} 115$ chain are presented and discussed in some detail in Ref. [16]. Here, the particle and photon projections of the $4^{\text {th }}$ generation $\alpha$-decay step ${ }^{286} \mathrm{Mt} \rightarrow{ }^{272} \mathrm{Bh}$, are re-iterated in the top row of Fig. 4. Our experimental information goes, however, beyond simple particle or photon singles spectra. The measurements include particle-photon and photon-photon correlations. Note that 'particle' always denotes either an energy release of a single $\alpha$ particle, or the sum of energies released by an $\alpha$ particle combined with those of coincident conversion or Auger electrons related to electromagnetic decays of excited states in the daughter nucleus. Specifically, in such more complex scenarios, the opportunity for self-consistent cross checks based on virtual high-statistics experiments with a Geant4-based version of TASISpec is rewarding [16, 27]. Based on a given decay scenario, the Geant4 simulations readily take care of such possible energy summing in the silicon detectors. Moreover, besides the above mentioned experimental particle and photon singles spectra, the simulations, hence the presumed decay scenarios, must also yield or account for the observed correlations, namely [16]:

- a particle-photon coincidence 9.53(1) MeV-434(1) keV,

- a particle-photon coincidence 9.60(1) MeV-362(1) keV,

- a particle-photon coincidence escape- $\alpha-362(1) \mathrm{keV}$,

- a particle-photon coincidence 9.58(1) MeV-303(1) keV,

- a particle-photon coincidence 9.65(1) MeV-232(1) keV,

- a particle-photon coincidence 9.68(4) MeV-172(1) keV,

- a photon-photon coincidence $136(1) \mathrm{keV}-167(1) \mathrm{keV}$ observed in two different Ge-detectors of the four Ge-detectors positioned upstream.

Rows 2 to 4 in Fig. 4 provide the normalized results of the simulations of 100000 decays of ${ }^{276} \mathrm{Mt}$ based on the respective decay scenario (i), (ii), or (iii) presented on the left-hand side. The spectra in columns (a) and (d) 
(a)

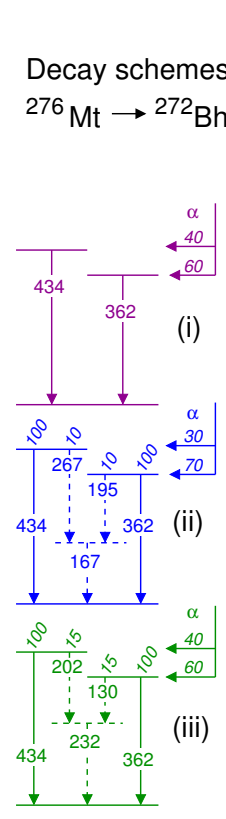

(b)

(c)

(d)

(e)

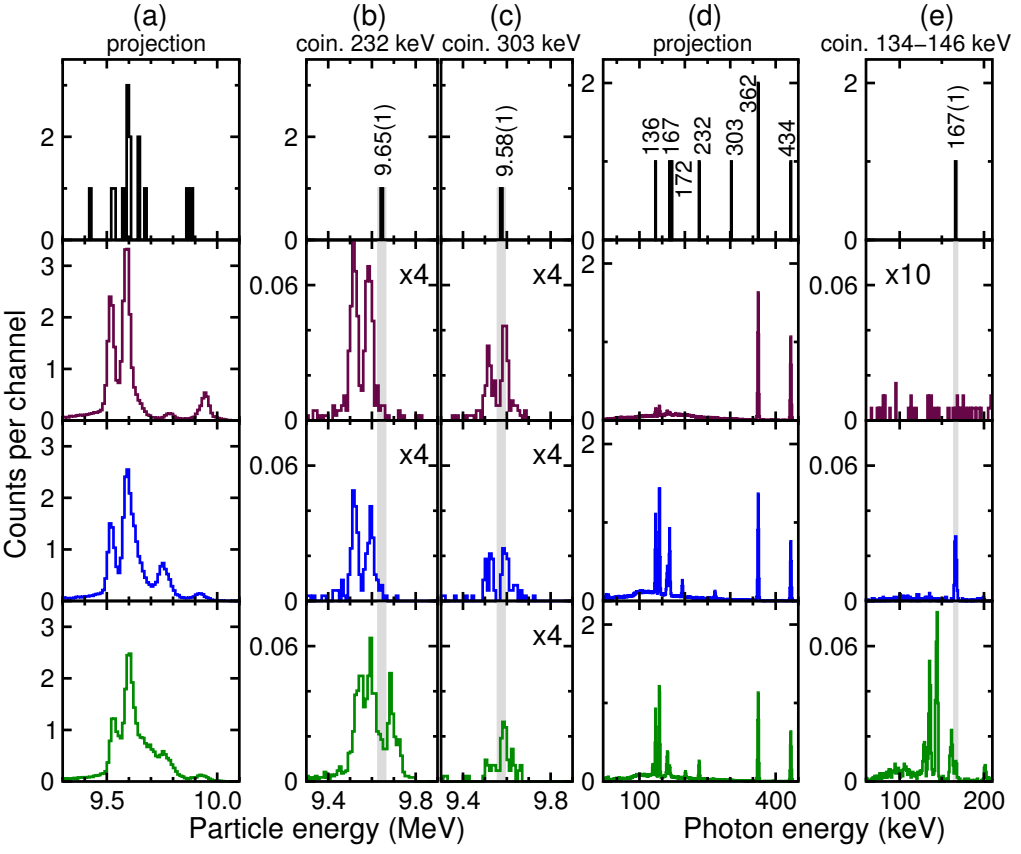

Fig. 4. Color on-line: Experimental (top row) and normalized simulated [27] particle and photon spectra based on presumed decay schemes of the $4^{\text {th }}$-generation ${ }^{288} 115$ decay step ${ }^{276} \mathrm{Mt} \rightarrow{ }^{272} \mathrm{Bh}$. From left to right, the columns provide the decay schemes, the total particle spectra, coincidence spectra with [230,234]-keV and $[301,305]-\mathrm{keV}$ photons, the total photon spectrum, and a photon spectrum in coincidence with ${ }^{272} \mathrm{Bh} K_{\alpha} \mathrm{X}$ rays, i.e. with $[134,146]-\mathrm{keV}$ photons.

show the projected particle and photon spectra similar to Fig. 2 in Ref. [16]. Columns (b) and (c) are particle spectra measured and expected in coincidence with $232-\mathrm{keV}$ and $303-\mathrm{keV}$ photons, while the rightmost column (e) shows photon spectra in coincidence with $\mathrm{Bh} K_{\alpha} \mathrm{X}$ rays.

Decay scenario (i) considers only the established skeleton of the ${ }^{276} \mathrm{Mt}$ decay, namely the 9.53(1)-MeV-434(1)-keV and 9.60(1)-MeV-362(1)-keV branches. Notably, the particle projection above $9.60 \mathrm{MeV}$ is difficult to describe, which becomes more apparent in the case of the $232-\mathrm{keV}$ coincidence requirement. The $303-\mathrm{keV}$ photon is readily explained as a Compton scattered $362-\mathrm{keV} \gamma$ ray. Unless one of them being of random origin, the 136(1)-167(1) photon-photon coincidence in two different upstream Gedetectors is difficult to be reconciled with the simple decay scheme: Since $136+167<362<434$, it could only be explained by two Compton scatterings, the probability of which is of the order of only $10^{-4}-10^{-5}$. In turn, this coincidence calls for the inclusion of an intermediate level at about 
$200 \mathrm{keV}$ [16]. For example, its existence would open the possibility for a coincidence between a $\gamma$ ray at $167 \mathrm{keV}$ and a $\mathrm{Bh} K_{\alpha} \mathrm{X}$ ray at $136 \mathrm{keV}$ [scenario (ii)], or for the observation of coincident $\mathrm{Bh} K_{\alpha}$ and $K_{\beta} \mathrm{X}$ rays at 136 and $167 \mathrm{keV}$, respectively. This scenario (iii) yields proper particle projections and particle-photon coincidence spectra. However, it is hampered by the lack of observing the $K_{\alpha}-K_{\alpha}$ coincidence favored by the simulations. The 136-167-keV coincidence is nicely accounted for by scenario (ii), but here the observed 9.65(1)-MeV-232(1)-keV correlation remains critical. These and further options will be detailed in Ref. [25]. Clearly, more experimental high-quality particle-photon coincidence data is necessary to settle the tentative center of the decay scheme of ${ }^{276} \mathrm{Mt}$. In any case, the phase space of some $2 \times 180 \mathrm{keV}=360 \mathrm{keV}$ required for the coincident observation of two $\mathrm{Bh} K \mathrm{X}$ rays is made available by the $362(1)$ and $434(1)-\mathrm{keV} \gamma$ rays.

\section{Conclusions}

"Thirty correlated $\alpha$-decay chains were observed following the reaction ${ }^{48} \mathrm{Ca}+{ }^{243} \mathrm{Am}$. Decay schemes arising from high-resolution spectroscopic coincidence data, in conjunction with comprehensive Monte-Carlo simulations, open the door for direct nuclear structure insights" [16] including candidates for $Z$-fingerprinting via characteristic $K \mathrm{X}$ rays. "Previous assignments linking the majority of the decay chains to the decay of 287,288115 [17, 18] are confirmed" [16]. Besides, we have also observed 7 decay chains with fewer members. Though similar to 4 such chains collected in Ref. [18], their interpretation indicates a more complex picture of the decay properties of ${ }^{289} 115$ and, consequently, ${ }^{293} 117$ [3]. More thorough statistical analyses as well as more detailed nuclear structure and decay assessments are underway [25].

The authors would like to thank the ion-source and accelerator staff at the GSI. This work is supported by the European Community FP7 Capacities ENSAR No. 262010, the Royal Physiographic Society in Lund, the Euroball Owners Committee, the Swedish Research Council, the German BMBF, the Office of Nuclear Physics, U.S. Department of Energy, and the UK Science and Technology Facilities Council.

\section{REFERENCES}

[1] S.G. Nilsson et al., Nucl. Phys. A131, 1 (1969).

[2] Y. Oganessian, J. Phys. G 34, R165 (2007).

[3] Yu.Ts. Oganessian et al., Phys. Rev. C87, 054621 (2013).

[4] Yu.Ts. Oganessian, Radiochim. Acta 99, 429 (2011). 
[5] S. Hofmann et al., Eur. Phys. J. A32, 251 (2007).

[6] L. Stavsetra et al., Phys. Rev. Lett. 103, 132502 (2009).

[7] R. Eichler et al., Radiochim. Acta 98, 133 (2010).

[8] P.A. Ellison et al., Phys. Rev. Lett. 105, 182701 (2010).

[9] Ch.E. Düllmann et al., Phys. Rev. Lett. 104, 252701 (2010).

[10] J.M. Gates et al., Phys. Rev. C83, 054618 (2011).

[11] S. Hofmann et al., Eur. Phys. J. A48, 62 (2012).

[12] R.C. Barber et al., Pure Appl. Chem. 83, 1485 (2011).

[13] G.T. Seaborg, W.D. Loveland, The Elements Beyond Uranium, Wiley-Interscience, New York 1990.

[14] R.-D. Herzberg, P.T. Greenlees, Prog. Part. Nucl. Phys. 61, 674 (2008).

[15] C.E. Bemis et al., Phys. Rev. Lett. 31, 647 (1973).

[16] D. Rudolph et al., Phys. Rev. Lett. 111, 112502 (2013).

[17] Yu.Ts. Oganessian et al., Phys. Rev. C69, 021601(R) (2004).

[18] Yu.Ts. Oganessian et al., Phys. Rev. C87, 014302 (2013).

[19] M. Schädel, Eur. Phys. J. D45, 67 (2007).

[20] A. Semchenkov et al., Nucl. Instrum. Methods B266, 4153 (2008).

[21] J. Runke et al., J. Radioanal. Nucl. Chem., DOI: 10.1007/s10967-013-2616-6.

[22] U. Forsberg et al., Acta Phys. Pol. B 43, 305 (2012).

[23] L.-L. Andersson et al., Nucl. Instrum. Methods A622, 164 (2010).

[24] U. Forsberg et al., Proceedings International Nuclear Physics Conference 2013, Florence, Italy, EPJ Web of Conferences, in press.

[25] U. Forsberg et al., to be published.

[26] K.-H. Schmidt, C.-C. Sahm, K. Pielenz, H.-G. Clerc, Z. Phys. A316, 19 (1984).

[27] L.G. Sarmiento, L.-L. Andersson, D. Rudolph, Nucl. Instrum. Methods A667, 26 (2012). 